\title{
Begriffe und Modelle der Überflutungsanalyse
}

\author{
Roman Maier · Stefan Reinstaller - Dirk Muschalla
}

Angenommen: 22. Februar 2021 / Online publiziert: 15. März 2021

(C) Der/die Autor(en) 2021

Zusammenfassung Niederschlagsinduzierte Überflutungen auf einer lokalen Skala erfahren in den letzten Jahren eine vermehrte Aufmerksamkeit. Vor allem im urbanen Raum ist in den letzten Jahren eine Vielzahl von Ereignissen mit erheblichen Schadenssummen aufgetreten. In der Fachdiskussion wird durch Klimawandel und Urbanisierung eine weitere Zunahme bezüglich Häufigkeit und Ausmaß dieser Ereignisse prognostiziert. Im deutschsprachigen Raum werden unterschiedliche Begriffe, teilweise in Abhängigkeit von den beteiligten Prozessen, benutzt. Neben der kurzen Darstellung der geläufigsten Fachbegriffe wird eine mögliche Klassifizierung vorgeschlagen. Für eine Überflutungsanalyse steht mittlerweile eine Vielzahl an ausgereiften Werkzeugen zur Verfügung. Eine hierarchische Darstellung der Modellansätze erlaubt eine der jeweiligen Aufgabe und dem jeweiligen Untersuchungsgebiet angepasste Auswahl der Modellansätze und eventuelle Kombination dieser. Dabei wird auf GIS-basierte Methoden zur Bestimmung der Fließwege an der Oberfläche, Zelluläre Automaten zur Bestimmung von Überflutungsflächen, 1D-hydrodynamische Kanalnetzmodelle, 2D-hydrodynamische Oberflächenabflussmodelle und gekoppelte 1D-2DÜberflutungsmodelle eingegangen.

Schlüsselwörter Pluviale Hochwässer . Hangwasser · Modellkonzepte . Überflutungsklassifizierung

DI R. Maier, BSc .

DI S. Reinstaller, BSc .

Univ.-Prof. Dr.-Ing. D. Muschalla (ه)

Institut für Siedlungswasserwirtschaft und Landschaftswasserbau,

Technische Universität Graz,

Streymayrgasse 10/I, 8010 Graz,

Österreich

d.muschalla@tugraz.at

\section{Flooding analysis - terms and models}

Abstract Stormwater induced flooding on a local scale has received increased attention in recent years. Especially in urban areas, a large number of events with considerable amounts of damage have been reported in the last few years. In the expert discussion, a further increase in the frequency and magnitude of these events is predicted due to climate change and urbanization. In the German-speaking world, different terms are used, partly depending on the processes involved. In addition to a brief description of the most common technical terms, a possible classification is proposed. In the meantime, a large number of sophisticated tools are available for a flood analysis. A hierarchical presentation of the model approaches allows a selection of the model approaches and possible combination of them adapted to the specific task and the respective study area. GISbased methods for determining surface flow paths, cellular automata for determining floodplains, 1D hydrodynamic sewer models, 2D hydrodynamic surface runoff models, and coupled 1D-2D flooding models are discussed.

Keywords Pluvial flooding · Surface water · Model concepts · Flood classification

\section{Hintergrund}

Überflutungen können weltweit auftreten und gehören $\mathrm{zu}$ jenen Naturgefahren mit dem höchsten Schadenspotenzial. Der globale wirtschaftliche Schaden aufgrund von Überflutungen wird in der Zeitspanne von 1980 bis 2013 auf eine Billion Dollar geschätzt (Berndtsson et al. 2019; Winsemius et al. 2016). Überflutungsmodelle sind mittlerweile ein häufig angewandtes Werkzeug in der Praxis, um die Überflutungsflächen aufgrund von Starkniederschlägen in einem Einzugsgebiet zu bestimmen. Aus den Ergebnissen können in weiterer Folge geeignete Reduk- tionsmaßnahmen abgeleitet werden, um mit der steigenden Problematik von Überflutungen umzugehen.

Einzelne Überflutungsereignisse entstehen durch unterschiedliche Ursachen, woraus in Folge unterschiedliche Arten beziehungsweise Kategorien von Überflutungen resultieren. Die Differenzierung dieser Überflutungskategorien erscheint auf den ersten Blick zwar trivial, dennoch existiert weltweit wie auch im deutschsprachigen Raum kein einheitliches Verständnis oder eine abgestimmte Definition bezüglich der verschiedenen Überflutungsarten. Der in Österreich gegenwärtig häufig benutzte der Begriff des Hangwassers wird teilweise mit dem internationalen Begriff der pluvialen Überflutung gleichgesetzt wird (RMP 2015), aber auch als (pluviale) Sturzflut oder als Oberflächenabfluss bezeichnet, in der hydrologischen Literatur ist dieser als der laterale Zwischenabfluss in der oberen Bodenzone definiert (Chifflard et al. 2008). Zahnt et al. (2018) wiederum differenzieren zwischen fluvialen und pluvialen Hochwässern, wobei das pluviale Hochwasser nochmals detaillierter in Oberflächenwasser, Überlastung des Entwässerungssystems und Grundwasser unterteilt wurde. Zekâi (2017) unterscheidet wiederum die Art der Überflutung in einer ersten Grobeinteilung wie folgt: i) gewöhnliche Überflutungen (Überflutungen, welche auf hydrologischen, meteorologischen und geomorphologischen Ereignissen basieren, z.B. Fluss-Hochwasser); ii) Sturzfluten. Im nationalen Kontext wird vielfach zwischen fluvialen (Wasser aus einem Fließgewässer) und pluvialen Hochwässern (Wasser, welches nicht aus einem Fließgewässer stammt) differenziert. Ebenfalls muss aufgrund der topografischen und meteorologischen Gegebenheiten in Österreich eine mögliche Schmelzwasserproblematik im Überflutungskontext berücksichtigt werden. Diese Einteilungen beschränken sich jedoch vorwiegend auf die Quelle und den daraus resultierenden Abfluss. Andere Faktoren wie die Niederschlagsart fließen nur am Rande in 
die Diskussion mit ein, sollten allerdings für eine exakte Kategorisierung mitberücksichtigt werden. Daher ist eine exakte Einteilung bezüglich der Überflutungskategorien mit allen relevanten Faktoren notwendig, um die relevanten Prozesse für die jeweilige Überflutungsart identifizieren zu können.

Es wird deutlich, dass je nach Fachgebiet oftmals dieselben Definitionen für verschiedene Überflutungsarten verwendet werden. In urbanen Einzugsgebieten wird die Kategorisierung zusätzlich erschwert, da ein urbanes Überflutungsereignis aufgrund einer Kombination von mehreren Überflutungsquellen und Kategorien auftreten kann. Eine Überflutung kann aufgrund des Erreichens der hydraulischen Kapazitätsgrenze der Kanalisation oder des Fließgewässers entstehen. Zusätzlich kann die Situation aufgrund eines auftretenden Hangabflusses aus einem naheliegenden Außengebiet beeinflusst werden. Es treten häufig Kombinationen aus pluvialen und fluvialen Überflutungen in urbanen Einzugsgebieten auf (Kron et al. 2019).

Diese Vielzahl an möglichen Prozesskombinationen beeinflusst maßgeblich die Auswahl der Prozesse, die in einem Simulationsmodell zur Analyse von urbanen Überflutungen und Hangwasser berücksichtig werden müssen und hat daher auch zwangsläufig Einfluss auf die Modellstruktur des Überflutungsmodells. Neben der räumlichen Struktur des Einzugsgebiets wird die Auswahl der Prozesse zusätzlich durch den betrachteten Niederschlagstyp beeinflusst. Eventuell können einzelne Teilprozesse vernachlässigt werden. Beispielweise sind der laterale Zwischenabfluss und die Verdunstung bei einer Sturzflut aufgrund der kurzen Dauerstufen des Ereignisses zu vernachlässigen (Mosley and McKerchar 1993). Bei Niederschlagsereignissen mit langer Dauerstufen ist eine solche Vernachlässigung nicht zulässig. Zusammenfassend sind also die räumliche Struktur des Einzugsgebietes sowie der für die Aufgabenstellung relevante Niederschlagstyp die entscheidende Randbedingung für die Klassifizierung der Überflutungen und der daraus abzuleitenden Modellansätze und -struktur.

Obwohl die Prozesse häufig auf denselben physikalischen Grundsätzen basieren, muss die Modellstruktur in Abhängigkeit von der Überflutungsart gewählt werden, sodass alle abflussre- levanten Prozesse im Modell enthalten sind. In der Praxis zeigt sich allerdings, dass häufig Modelle zur Anwendung kommen, welche zwar für eine Überflutungsart geeignet sind, jedoch nicht auf alle Aufgabenstellungen im Kontext der Überflutungsmodellierung übertragbar sind. Beispielweise ist das 2DModell von fluvialen Überflutungen nicht in derselben Form auf urbane
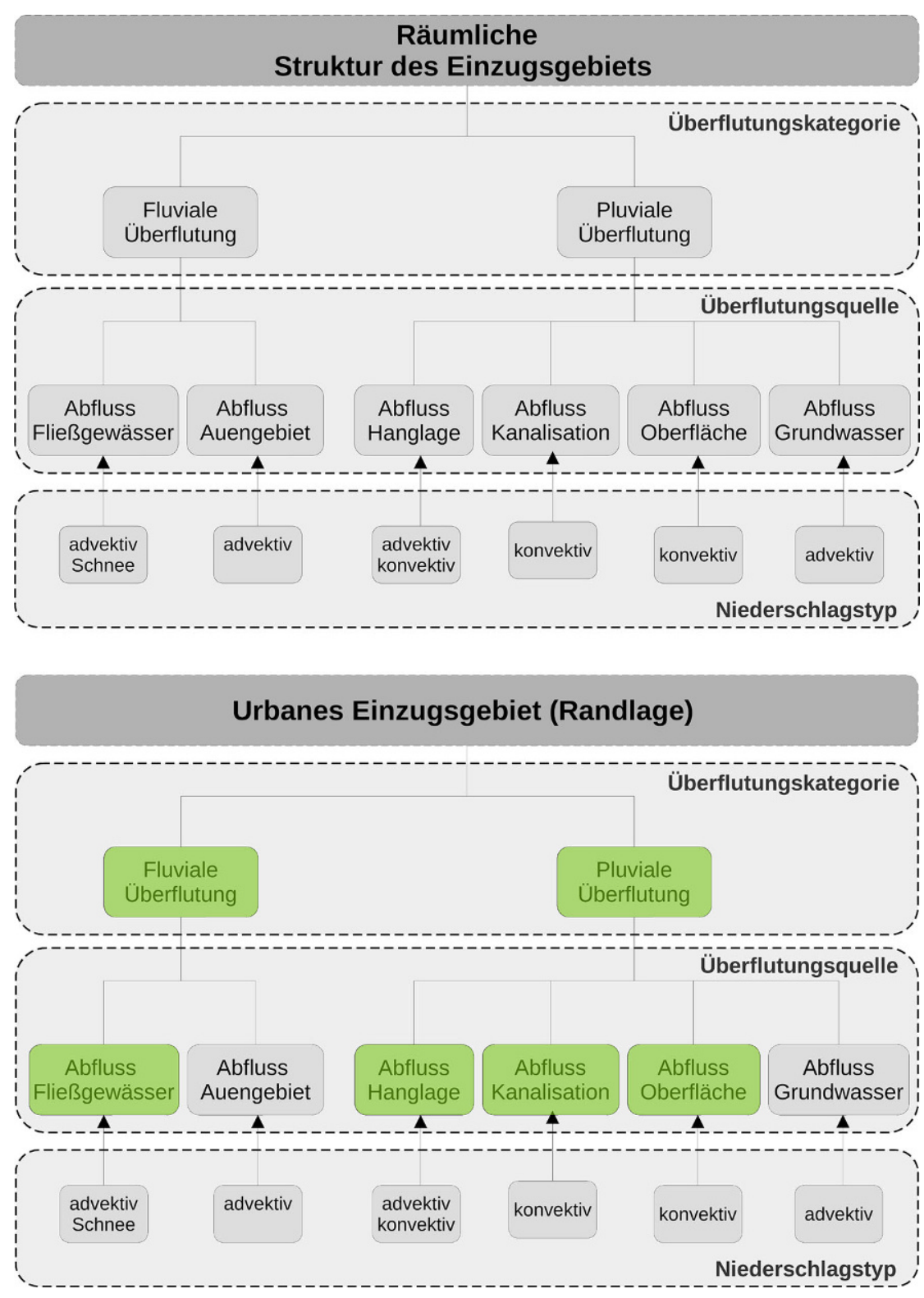

Abb. 1 Klassifizierung von Überflutungen mit den 4 Hauptfaktoren Räumliche Struktur, Hauptkategorie der Überflutung, daraus resultierende mögliche Quellen in Form von Abflüssen und die Zuweisung des maßgeblichen Niederschlagstyps; oben: allgemeine Einteilung für jede mögliche Struktur eines Einzugsgebiets, unten: exemplarisches Beispiel für ein urbanes Einzugsgebiet am Stadtrand (grün markierte Stellen symbolisieren die relevanten Überflutungsquellen mit der Zuordnung zu der jeweiligen Hauptkategorie) 
stellt. In weiterer Folge werden die unterschiedlichen Modellierungsansätze der urbanen Überflutungsmodellierung beschrieben sowie deren Stärken und Schwächen hinsichtlich der Anwendbarkeit auf die unterschiedlichen Arten der Überflutungen diskutiert.

\section{Klassifizierung von Überflutungen}

Allgemein kann von Überflutungen gesprochen werden, wenn eine Oberfläche temporär vollständig mit Wasser bedeckt ist, welche im üblichen $\mathrm{Zu}$ stand frei von Wasser ist (EC 2007). Dieser Zustand wird durch verschiedene Faktoren beeinflusst. Der erste Faktor ist der Typ der räumlichen Struktur des Einzugsgebiets. Dazu werden vier Hauptstrukturen definiert, welche in Österreich am häufigsten vorkommen: i) urbane Innenstadt; ii) urbane Randlage, iii) ländliche Kleinstadt; iv) rural - nicht besiedeltes Einzugsgebiet. Je nach strukturellen Eigenschaften des Einzugsgebiets wird die grobe Überflutungskategorie (fluvial und/ oder pluvial) bestimmt, wobei auch die Kombination der beiden Kategorien möglich ist. Auf Basis der Überflutungskategorien werden die einzelnen Überflutungsquellen im Einzugsgebiet definiert. Auf diesen lassen sich die einzelnen abflussrelevanteren Pro- zesse ableiten, welche die Grundlage für die Modellstruktur sind. In einem letzten Schritt werden den einzelnen Überflutungsquellen die maßgeblichen Niederschlagstypen zugewiesen. In diesem Zusammenhang wird zwischen kurzzeitig-intensiven und lokal auftretenden Niederschlägen (konvektiv) und langanhaltenden-konstanten Niederschlägen (advektiv) unterschieden. Durch die alpingeprägte Topografie in Österreich kann es auch zu Überflutungen aufgrund einer saisonalen Schneeschmelze kommen, welche vor allem in ländlichen (ruralen) Einzugsgebieten in Österreich zu Überflutungsproblemen führen. Daraus resultieren vier entscheidende Faktoren zur Klassifizierung von Überflutungen (Abb. 1): i) Überflutungskategorie, ii) Überflutungsquelle, iii) räumliche Struktur des Einzugsgebiets und iv) Niederschlagstyp.

\section{Allgemeines \\ Modellierungskonzept eines Überflutungsmodells}

Der dominierende Prozess bezüglich Überflutungen ist der Oberflächenabfluss. Da dieser vor allem vom Niederschlagsvolumen abhängig ist, wird in der Hydrologie oftmals von Niederschlags-Abfluss-Modellen gesprochen. Diese Definition verdeutlicht bereits den Zusammenhang zwischen hydrologischer Abflussbildung und dem hydraulischen Fließverhalten an der Oberfläche im Überflutungsfall. Die hydrologische Abflussbildung ist im Allgemeinen eine Bilanz aus den hydrologischen Prozessen Niederschlag, Verdunstung, Transpiration, Interzeption, Infiltration, Speicherverlusten, Zwischenabfluss und Grundwasserneubildung (Sitterson et al. 2017).

Diese Prozesse können je nach Aufgabenstellung und räumlich struktureller Eigenschaften des Einzugsgebiets vernachlässigt werden. Ist eine Hanglage innerhalb des Einzugsgebiets vorhanden, muss dies im Zuge der hydrologischen Modellparameter bereits in der Abflussbildung berücksichtigt werden. Die Eingangsgröße ist dabei das Niederschlagsvolumen, welches sowohl eine zeitliche als auch eine räumliche Abhängigkeit aufweist. Das Modellergebnis ist jenes Volumen, welches an der Oberfläche zum Abfluss kommt und als Oberflächenabfluss bezeichnet wird.

Die räumliche Ausbreitung des Oberflächenabflusses sowie der zeitliche Verlauf der Abflusswelle werden anhand des Oberflächenabflussmodells berechnet. Je nach gegebener Struktur des Einzugsgebiets müssen zusätzliche Überflutungsquellen wie ein mögliches Überstauvolumen aus der Kanalisa-

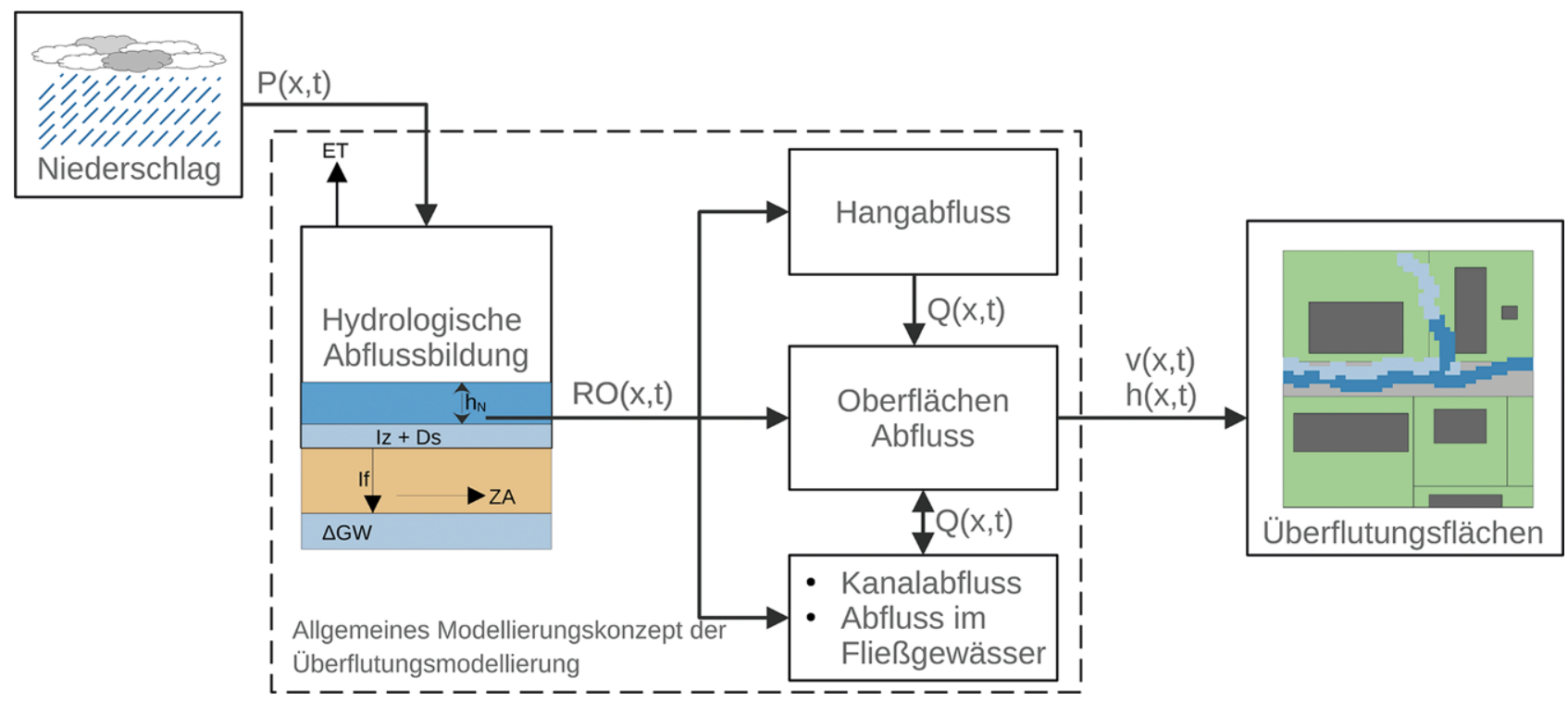

Abb. 2 Allgemeines Modellkonzept eines Überflutungsmodells zur Bestimmung der Überflutungsflächen (Zielgrößen: Geschwindigkeitsvektor $(\mathrm{v}(\mathrm{x}, \mathrm{t}))$ und Wasserstand $(\mathrm{h}(\mathrm{x}, \mathrm{t}))$ basierend auf der hydrologischen Abflussbildung (Niederschlag $(\mathrm{P}(\mathrm{x}, \mathrm{t}))$, Verdunstung und Transpiration (ET), Oberflächenabfluss ( $R O(x, t)$ ), Interzeption (Iz), Muldenverluste (Ds), Infiltration (If), laterale Zwischenabfluss $(Z A)$, Grundwasseranreicherung $(\Delta G W)$ ) und der räumlichen und zeitlichen Ausbreitung des Oberflächenabflusses (beeinflusst durch einen angrenzenden Hangabfluss sowie einem Abfluss aus der Kanalisation und eines Fließgewässers $(\mathrm{Q}(\mathrm{x}, \mathrm{t}))))$ 
tion sowie eines Fließgewässers berücksichtigt werden. Zusätzlich kann ein Hangabfluss aus einer naheliegenden Hanglage das Abflussvolumen an der Oberfläche erhöhen. Das Ergebnis des Oberflächenabflussmodells sind die Fließwege und Überflutungsflächen an der Oberfläche, welche durch den resultierenden Wasserstand und Geschwindigkeitsvektoren dargestellt werden können. Das Modellergebnis sind daher Wasserstand, Fließgeschwindigkeit sowie Fließrichtung. Zusammenfassend besteht ein Überflutungsmodell grob aus zwei Teilmodellen: hydrologisches Modell zur Abflussbildung und Oberflächenabflussmodell zur Bestimmung des Fließwegs und der Überflutungsflächen, welche unidirektional miteinander gekoppelt sind (Abb. 2).

\section{Modellierungsansätze zur Bestimmung der Fließwege und Überflutungsflächen an der Oberfläche}

Es existiert eine Vielzahl an unterschiedlichen Modellen bezüglich Überflutungen, welche sowohl kommerziell vertrieben werden als auch offen als Open-source- oder Freewareprodukt verfügbar sind. Die Modellierungsansätze reichen dabei von einfachen Ansätzen mit geringem Datenaufwand und kurzen Rechenzeiten (z.B. GISbasierte D8-Methode zur Fließwegbestimmung) bis hin $\mathrm{zu}$ komplexen Ansätzen mit enormen Datenmengen sowie langen Simulationszeiten (z.B. hydrodynamische integrierte 1D-2DModelle). In Abhängigkeit von den gesuchten Zielgrößen muss ein passendes Modell gewählt werden, welches je nach Modellansatz variieren kann (Fließweg an der Oberfläche, Überflutungsflächen sowie Überstauvolumen).

Um ein geeignetes Modell für eine wasserwirtschaftliche Fragestellung im Überflutungskontext auszuwählen, bedarf es mehrerer Faktoren als Entscheidungsgrundlage. Neben der Modellkomplexität, den notwendigen Daten und den erwarteten Simulationszeiten sind auch die gesuchten Zielgrößen des Modells ein entscheidender Faktor für die Auswahl (Abb. 3).

\subsection{GIS-basierte D8-Methode zur} Bestimmung der Fließwege an der Oberfläche

Die D8-Methode (O'Callaghan and Mark 1984; Jenson and Domingue 1988;

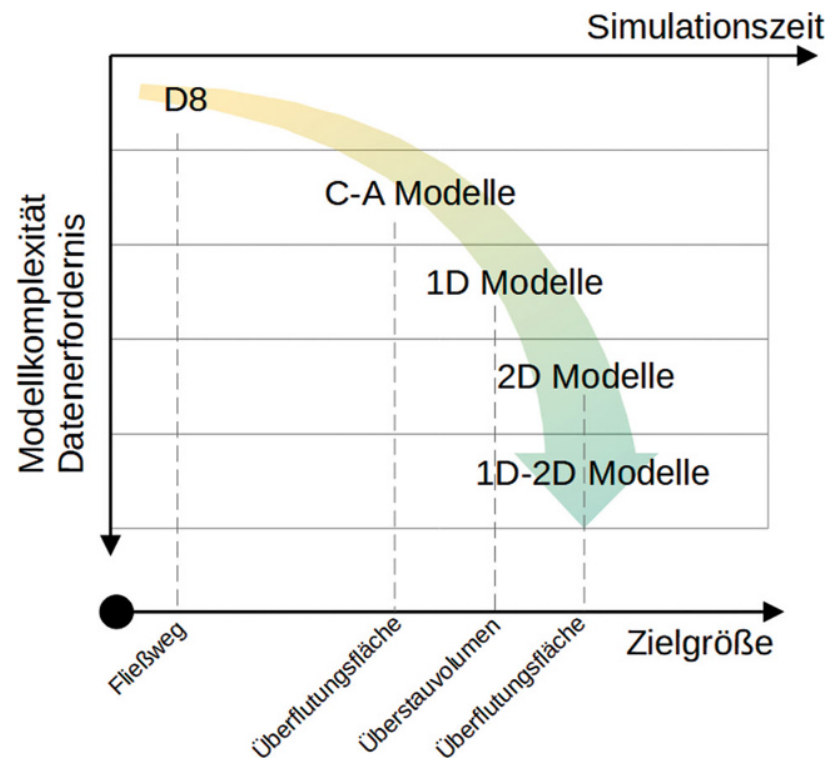

Abb. 3 Hierarchischgeordnete ModellansätzederÜberflutungsmodellierung basierend auf vier Faktoren der Modellauswahl (Modellkomplexität, Datenerfordernis, Simulationszeit und ZielgröBen)

Fairfield and Leymarie 1991) kann anhand eines digitalen Geländemodells (DGM) angewandt werden. Dabei wird die räumliche Höheninformation anhand einer GIS-Software verarbeitet, um daraus Fließwege und eine Abflussakkumulation berechnen $\mathrm{zu}$ können.

Abb. 4 zeigt die Funktionsweise der D8-Methode. Es wird angenommen, dass jede Rasterzelle von acht Nachbarzellen umschlossen wird und dieser Zelle aufgrund ihrer und der benachbarten Zellhöhen eine Abflussrichtung zugeteilt wird (mittlere Bilder in Abb. 4). Kann aufgrund der Höhensituation kein Abfluss stattfinden, wird von einer Senke gesprochen. Durch die D8Methode wird somit für jede Zelle innerhalb des Rasters die Abflussrichtung bestimmt, womit sich schlussendlich ein Oberflächenabfluss akkumulieren lässt (Anzahl der Zellen, die in eine bestimmte Zelle fließen - letztes Bild in Abb. 4). Durch die Kombination der gewonnenen Informationen lässt sich ein Oberflächenentwässerungssystem ermitteln.

Die Qualität des Berechnungsergebnisses hängt dabei von der Auflösung des DGM und von etwaigen notwendigen Anpassungen des verwendeten Modells ab, dabei gilt grundsätzlich: je höher die Auflösung des DGM, desto besser die Ergebnisse. Vor allem im urbanen Bereich ist eine hohe Auflösung essenziell, um brauchbare Abflussakkumulationen berechnen zu können. Ebenso müssen in diesem Bereich sowohl die Bebauung (wie etwa Gebäude als Abflusshindernisse) und etwaige Besonderheiten der Infrastruktur (z. B. Unterführungen) als auch auftretende Senken berücksichtigt werden, um korrekte Ergebnisse zu generieren.

Die D8-Methode allein eignet sich vor allem dafür, einen ersten, aber wichtigen Überblick über ein Einzugsgebiet zu erhalten. Für die Analyse wird nur ein Oberflächenabfluss angenommen, die Abflussbildung selbst wird nicht berücksichtigt. Da diese Methode schnell und einfach anzuwenden ist und DGM-Daten in der Regel in ausreichender Auflösung verfügbar sind, können erste kritische Punkte in Untersuchungsgebieten bereits identifiziert werden. Der Nachteil dieser Methode ist, dass dynamische Abflüsse und damit auftretende Effekte wie Rückstau und daraus resultierende etwaige Fließumkehrungen nicht abgebildet werden können. Die D8-Methode kann als Grundlage für komplexere Oberflächenabflussmodelle dienen, um neuralgische Punkte im Betrachtungsgebiet $\mathrm{zu}$ identifizieren und die $\mathrm{zu}$ berücksichtigenden Einzugsgebietsgrenzen zu bestimmen. Die Methode wird auch angewandt, um Fließpfadkarten zur Darstellung der theoretischen Fließwege des Oberflächenabflusses bzw. 


\begin{tabular}{|l|l|l|l|l|l|}
\hline 78 & 72 & 69 & 71 & 58 & 49 \\
\hline 74 & 67 & 56 & 49 & 46 & 50 \\
\hline 69 & 53 & 44 & 37 & 38 & 48 \\
\hline 64 & 58 & 55 & 22 & 31 & 24 \\
\hline 68 & 61 & 47 & 21 & 16 & 19 \\
\hline 74 & 53 & 34 & 12 & 11 & 12 \\
\hline
\end{tabular}

Digitales Höhenmodell

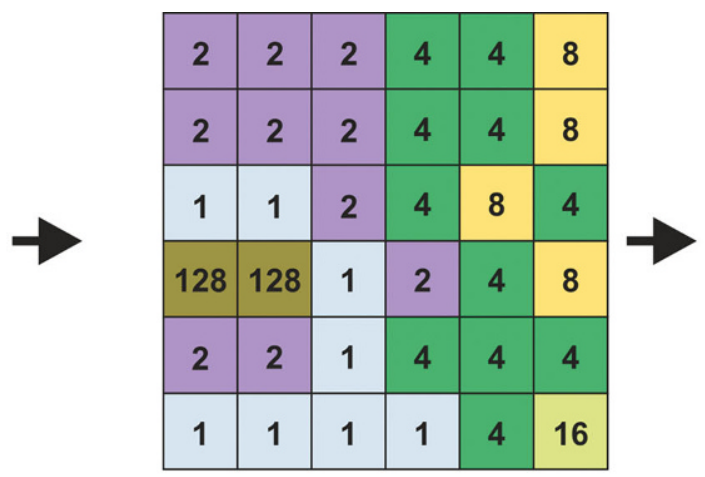

Fließrichtung

\begin{tabular}{|l|l|l|l|l|l|}
\hline 0 & 0 & 0 & 0 & 0 & 0 \\
\hline 0 & 1 & 1 & 2 & 2 & 0 \\
\hline 0 & 3 & 7 & 5 & 4 & 0 \\
\hline 0 & 0 & 0 & 20 & 0 & 1 \\
\hline 0 & 0 & 0 & 1 & 24 & 0 \\
\hline 0 & 2 & 4 & 7 & 35 & 1 \\
\hline
\end{tabular}

Abflussakkumulation

\begin{tabular}{|c|c|c|}
\hline 32 & 64 & 128 \\
\hline 16 & & 1 \\
\hline 8 & 4 & 2 \\
\hline
\end{tabular}

Abb. 4 Veranschaulichung der D8-Methode-Fließrichtung bzw. Abflussakkumulation. (ESRI 2021 mod.)

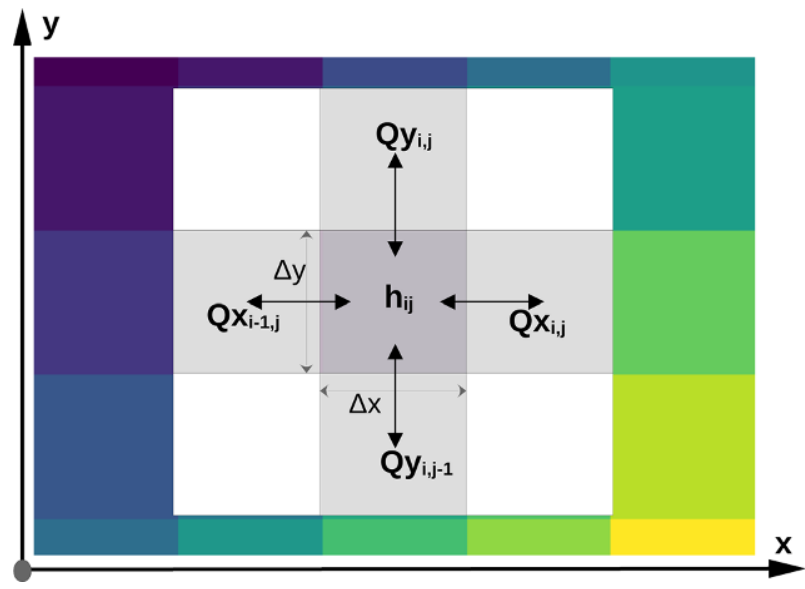

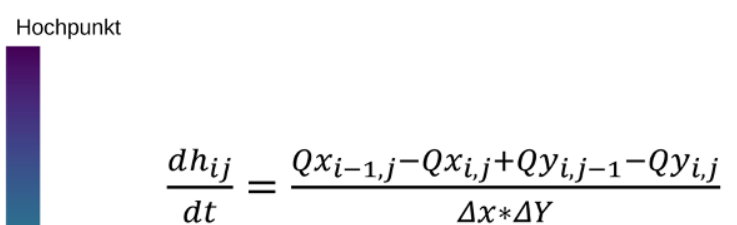

DGM

$$
Q x_{i, j}=\frac{d_{f l o w}^{5 / 3}}{n} *\left(\frac{h_{i-1, j}-h_{i, j}}{\Delta x}\right)^{1 / 2}
$$

Abb. 5 Beispielhafte Ausführung eines Zellulären Automaten zur Bestimmung des Wasserstands $\left(h_{i j}\right)$ in Zelle $e_{i j}$ und einer gegebenen Zellenauflösung in Abhängigkeit von der Auflösung des digitalen Geländemodells. Berechnung des Wasserstands auf Basis der Kontinuitätsgleichung (GL-1) und der kinematischen Wellengleichung (GL-2) zur Ermittlung des Durchflusses von Zelle zu Zelle, mit $\mathrm{d}_{\mathrm{flow}}=$ Fließtiefe $z$ wischen zwei Zellen, $n=$ Manning-Rauheit und $\mathrm{Q}=$ Volumenströme zwischen zwei Zellen. (Mod. Bates und De Roo 2000)

Hangwassers zu erstellen, wie sie in Österreich mittlerweile vielfach auf den entsprechenden Portalen der Bundesländer zu finden sind.

\subsection{Zelluläre Automaten zur Bestimmung von Überflutungsflächen}

Überflutungen sind ein räumlich diskreter und dynamischer Vorgang. Daher kann der Modellansatz der zellulären Automaten für deren Modellierung angewendet werden. Mit diesem Ansatz wird die gesamte Oberfläche im Einzugsgebiet in ein Netz aus Rasterzellen mit einer festen Auflösung einge- teilt, welche in erster Linie von der Auflösung des verwendeten Digitalen Geländemodells (DGM) abhängig ist. Der Oberflächenabfluss wird anschließend in Abhängigkeit von der Höhenlage der Rasterzellen von Nachbarzelle zu Nachbarzelle dynamisch bestimmt (Abb. 5). Dabei wird der Oberflächenabfluss hydrodynamisch mit Lösung der entsprechenden physikalischen Gleichungen in stark vereinfachter Form (z.B. Manning-Strickler-Gleichung) abgebildet (LISFLOOD-FP, Bates und De Roo 2000). Modelle wie das CADDIES Model (Ghimire et al. 2013) kombinieren die Methodik zur Bestimmung der Fließwegrichtung (ähnlich der be- schriebenen D8-Methode) mit einer dynamischen Volumenbilanz zwischen den beiden betrachteten Rasterzellen. Über die Fläche der Rasterzelle kann im Anschluss der Wasserstand in der Rasterzelle dynamisch berechnet werden. Die Fließgeschwindigkeit wird anschließend über die Manning-StricklerGleichung berechnet. Kommerzielle Modelle wie FloodArea der geomer GmbH nutzen ähnliche Modellansätze zur Bestimmung des Wasserstands an der Oberfläche. Häufig werden dabei die abflussrelevanten hydrologischen Prozesse durch vereinfachte Ansätze berücksichtigt. Für die hydrologische Abflussbildung wird beispielweise häu- 
fig das Abflussbeiwertverfahren verwendet. Rein hydrologische Modelle wie LISFLOOD (Burek et al. 2013) nutzen dabei ebenfalls den Ansatz der zellulären Automaten, um die Abflussbildung detailliert pro Rasterzelle $\mathrm{zu}$ berechnen. Allerdings werden diese Modelle eher für wasserwirtschaftlichen Fragestellungen (Entstehung einer Hochwasserwelle) verwendet.

Ein großer Vorteil dieser Ansätze ist die gute Schnittstelle $\mathrm{zu}$ allen gängigen GIS-Software-Produkten, da zelluläre Automaten häufig das dasselbe Datenformat wie Rasterdaten in GISSystemen verwenden. Dadurch werden darüber hinaus die Visualisierung und die Kopplung mit anderen Modellen erleichtert. Durch die starken Vereinfachungen der abflussrelevanten Prozesse sind sehr schnelle Simulationszeiten zu erreichen. Der Datenaufwand (hochaufgelöstes DGM und Landnutzungsdaten als wichtigste Datengrundlage) ist moderat. Die starken Vereinfachungen hinsichtlich des Oberflächenabflusses müssen berücksichtigt werden, da diese je nach Gebietsstruktur zu ungenauen Ergebnissen führen können. Beispielweise werden abflussrelevante Rückstaueffekte und Fließumkehr häufig durch die Eliminierung der Beschleunigungsterme in den Grundgleichungen nicht berücksichtigt.

Die Anwendung von zellulären Automaten reicht von rein hydrologischen
Fragestellungen großer ruraler Einzugsgebiete zur Vorhersage von fluvialen Abflussganglinien bis hin zur Modellierung pluvialer und fluvialer Überflutungen im ländlichen und urbanen Raum. Durch die Abhängigkeit zur Auflösung der Rasterzelle ist es auch möglich, Modelle für größere Einzugsgebiete aufzubauen. Allerdings können pluviale Überflutungen aus der Kanalisation häufig nicht abgebildet werden, da der bidirektionale Austausch des Überstauvolumens zwischen Oberfläche und Kanal in den meisten Modellen nicht implementiert ist. Aus diesem Grund eignet sich dieser Modellansatz nur bedingt zur Modellierung von pluvialen Überflutungsereignissen in urbanen Einzugsgebieten. Jüngste Publikationen zu diesem Thema zeigen jedoch, dass durch eine Kopplung zweier Modelle auch Überflutungen in urbanen Einzugsgebieten modelliert werden können (z.B. Kopplung des 1D-Kanalmodells SWMM mit dem zellulären Automaten CA-FFé (Jamali et al. 2020)).

\subsection{D-Kanalmodell}

1D-Kanalabflussmodelle gibt es in verschiedenen Komplexitätsstufen. Mit den heute zur Verfügung stehenden Rechenkapazitäten werden hauptsächlich hydrodynamische Kanalnetzmodelle verwendet, die das vollständige de Saint Venant'sche Gleichungssystem lösen und somit Rückstau und damit auch Überstau während einer eventuellen hydraulischen Kanalnetzüberlastung abbilden. Bekannte Softwareprodukte, die im deutschsprachigen Raum häufig für solche Modelle Anwendung finden, sind neben anderen das Open-Source-Projekt SWMM5 der US EPA, Mike Urban von DHI, HystemExtran von ITWH und Kanal++ von AKG Software. Anhand des Kanalnetzkatasters und etwaiger notwendiger Informationen über Sonderbauwerke im Kanal und die Einleitungspunkte des Systems (Betriebe, Haushalte etc.) kann ein hydrodynamisches Kanalnetzmodell für eine Kommune aufgebaut werden (siehe dazu Muschalla et al. 2015). Zusammen mit einer Messkampagne an signifikanten Punkten des $\mathrm{zu}$ untersuchenden Systems sollte das Modell kalibriert und validiert werden, um damit im Anschluss realitätsnahe Szenarien analysieren zu können. Typische Ziele der Anwendung dieser Modellklasse sind die Nachweisführung verschiedener Belastungsfälle, die Optimierung der Kanalnetzsteuerung bis hin zur Anlagendimensionierung und Fremdwasseruntersuchung. Dabei ermöglichen heute verfügbare Rechenleistungen auch Langzeitsimulationen über Zeiträume von mehreren Jahren, bzw. gibt es bekannte Methoden, um entsprechende Anforderungen und

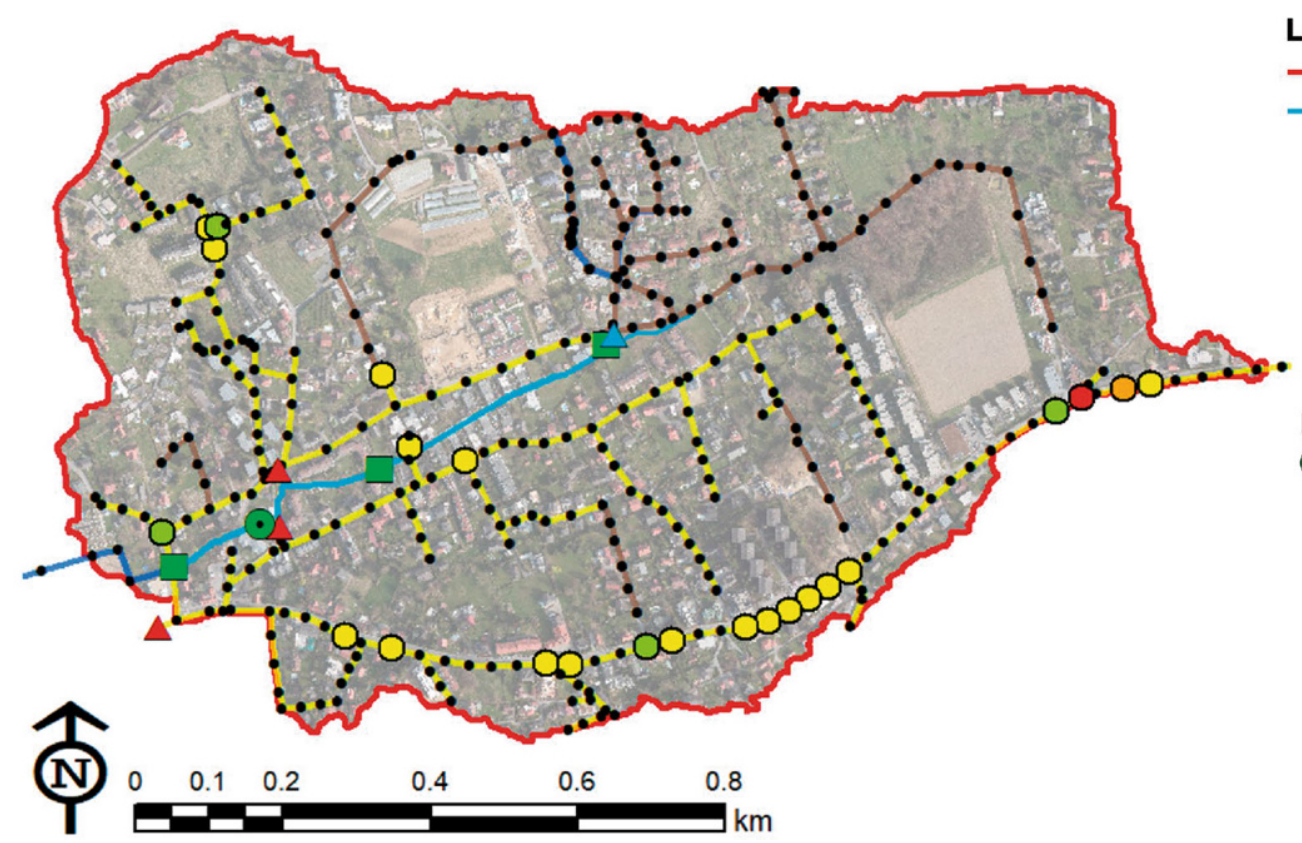

\section{Legende}

— Einzugsgebietsgrenze

- Bach

Kanalstrang

- Mischwasser

- Schmutzwasser

- Regenwasser

Systemauslauf

$\triangle$ Mischwasser

$\triangle$ Regenwasser

Speicherbauwerk

- Niederschlagsmessstation Überstauhäufigkeit eines Schachts in 10 Jahren
$0>3 \mathrm{mal}$
O $3 \mathrm{mal}$
O $2 \mathrm{mal}$
$1 \mathrm{mal}$
- kein Überstau

Abb. 6 Beispielergebnis eines Überstaunachweises ermittelt mit einer 10-jährigen Niederschlagsserie folgend der Methodik von Leimgruber et al. (2018) 
Fragestellungen effizient zu lösen, wie Leimgruber et al. (2018) demonstrieren.

Um nicht nur den Trockenwetterabfluss simulieren zu können, werden 1DKanalabflussmodelle in der Regel mit hydrologischen Modellen für die $\mathrm{Ab}$ flussbildung und den Oberflächenabfluss gekoppelt. Diese Kopplung erlaubt es, den Niederschlag über Abflussbildung und Abflusskonzentration zu berechnen und in das Kanalnetz einzuleiten, womit der Niederschlags-AbflussProzess für ganze Einzugsgebiete und eine hohe zeitliche und räumliche Auflösung simuliert werden können. Die in Österreich geforderten Nachweise über die Überstausicherheit eines Kanalsystems nach ÖWAV-Regelblatt 11 (2009), exemplarisch dargestellt in Abb. 6, oder den Mindestweiterleitungswirkungsgrad eines Mischwassersystems nach ÖWAV-Regelblatt 19 (2007) können damit durchgeführt werden. Mittlerweile können auch Maßnahmen zur Niederschlagswasserbewirtschaftung (oft als grün-blaue Infrastruktur bezeichnet) über das hydrologische Modell berücksichtigt werden, womit eine nachhaltige Niederschlagswasserbewirtschaftungsstrategie geplant und analysiert werden kann (Kleidorfer et al. 2019).

1D-Kanalnetzmodelle erlauben die Identifikation von hydraulischen Überlastungen des Kanalsystems und eine Ableitung der Überstauhäufigkeit von Kanalschächten. Dabei kann neben der Eintrittswahrscheinlichkeit auch das damit einhergehende Überstauvolumen bestimmt und bilanziert werden. Damit liefert das Modellergebnis wichtige Hinweise zur Identifikation von durch Überflutung gefährdeten Bereichen. Da die Ausbreitung des aus dem Kanalnetz austretenden Volumens an der Oberfläche jedoch nicht möglich ist, kann das Überflutungsrisiko nicht ermittelt werden.

Mit diesen Modellen ist es allerdings nicht möglich, direkte Aussagen zur Überflutungsgefahr zu treffen, da das aus dem Kanalnetz austretende Volumen an der Oberfläche zwar bilanziert wird, die Oberfläche selbst aber nur abstrahiert über das gekoppelte hydrologische Modell berücksichtigt ist. Eine Berechnung der Ausbreitung des aus dem Kanalnetz ausgetretenen Wassers auf den Oberflächen ist somit nicht möglich. Nichtsdestotrotz liefern ein hydrodynamisches Modell und ein mit diesem durchgeführter Überstaunachweis wichtige Hinweise zur Identifikation von durch ein überlastetes
Kanalnetz induzierte pluviale Überflutungen und eine wertvolle Grundlage für den Aufbau eines detaillierten 1D2D-Überflutungsmodells.

\subsection{D- und gekoppeltes 1D-2D- Überflutungsmodell}

Wie die Erfahrungen aus vergangenen Ereignissen gezeigt haben, treten Überflutungen häufig aufgrund einer Kombination mehrerer Ursachen auf (Sene 2015). Beispielweise kann das Entwässerungssystem aufgrund der erhöhten Belastung aus einem Starkniederschlagsereignis an seine Kapazitätsgrenzen kommen und den Oberflächenabfluss zusätzlich erhöhen. Gleichzeitig kann es zu einer Überlastung eines naheliegenden Fließgewässers kommen, welche durch einer Verklausung von Durchlässen zusätzlich verstärkt werden kann. Besonders in besiedelten Einzugsgebieten führen solche Kombinationswirkungen verstärkt zu Überflutungen mit hoher Schadensfolge (Zekâi 2017). Aus diesem Grund sollen die verwendeten Modellansätze alle möglichen abflussrelevanten Teilprozesse berücksichtigen, um die Vorhersagefähigkeit der Modelle zu erhöhen. Der gekoppelte 1D-2D-Modellansatz ist dabei in der Lage, diese Bedingungen großenteils $\mathrm{zu}$ erfüllen, sodass dieser für viele Fragestellung bezüglich der Überflutungsproblematik angewendet werden können.

Eine Anwendung dieses Ansatzes ist die Kombination von zweidimensionalem Oberflächenabfluss mit dem eindimensionalen Abflussverhalten der Kanalisation (Schmitt et al. 2004). Hierfür kommen 2D-hydrodynamische Oberflächenabflussmodelle, die zumeist die tiefengemittelten Flachwassergleichungen lösen, in Kombination mit den bereits beschriebenen 1D-hydrodynamischen Kanalnetzmodellen zur Anwendung. 2D-Oberflächenabflussmodelle sind ein seit Jahren in der (fluvialen) Überflutungsanalyse etablierter Standard, der die dynamische Berechnung von Wasserspiegellagen, Wasserständen und Fließgeschwindigkeiten ermöglicht und damit die Ermittlung aller relevanten Größen für eine Überflutungsanalyse erlaubt. Je nachdem, ob ein Einfluss des Kanalnetzes auf ein Überflutungsrisiko vorhanden ist, müssen die $2 \mathrm{D}$ hydrodynamischen Modelle mit einem entsprechenden Kanalnetzmodell gekoppelt werden. Der Einfluss der Kanalisation kann dabei einerseits durch aus dem Kanal austretendes Volumen (Überstau) oder durch eine über das Kanalnetz räumlich verlagerte Überflutung bestehen.

Ist eine gekoppelte Betrachtung notwendig, müssen daher die Wasserstände und Fließgeschwindigkeit auf der Oberfläche zeitsynchron mit einem möglichen Überstauvolumen aus der Kanalisation berechnet werden. Dabei werden die dynamischen Wellengleichungen in zwei- und eindimensionaler Form gelöst. Dieser Anwendungsfall wurde vielfach in verschiedensten urbanen Einzugsgebieten erfolgreich demonstriert (siehe dazu Leandro et al. 2009; Löwe et al. 2017; Fan et al. 2017). Bekannte kommerzielle Modelle, die im deutschsprachigen Raum für diese Aufgabe angewendet werden, sind MIKE FLOOD des Danish Hydraulic Institute (DHI) sowie HYSTEM EXTRAN 2D der itwh $\mathrm{GmbH}$.

In ähnlicher Form kann mittels des 1D-2D-Ansatzes auch die Überlastung eines Fließgewässers und dessen Ausbreitung auf der Oberfläche ermittelt werden. Hierbei wird der Abfluss im Fließgewässer eines Außengebiets eindimensional als äußere Randbedingung ermittelt und die Ausbreitung auf der Oberfläche zweidimensional gelöst (beispielhaft demonstriert in FinaudGuyot et al. 2011). Ebenfalls kann der Wasserstand eines stehenden Gewässers als äußere Randbedingung stromabwärts angesetzt werden (Pasquier et al. 2019), dies kann in Österreich beispielweise in Einzugsgebieten, die einen größeren See beinhalten, seine Anwendung finden. Ein verbreitertes Modell für diesen Anwendungsfall ist HEC-RAS in der Version 5.0 des Pionierkorps des Heeres der Vereinigten Staaten (USACE).

Da die Oberfläche des Einzugsgebiets durch ein zweidimensionales Netz des 2D-Modells räumlich aufgeteilt wird, kann der Ansatz gleicher hydrologischer Elemente (HRU) verwendet werden, um die hydrologische Abflussbildung bestimmen zu können (Leavesley et al. 1983). Jedem Element im Netz werden in Abhängigkeit von seiner Oberflächenbeschaffenheit (Landnutzung) hydrologische Parameter zugewiesen, wobei die notwendigen Parameter von den abflussrelevanten hydrologischen Teilprozessen sowie der räumlichen Struktur des Einzugsgebiets abhängig sind. Der entstehende Abfluss wird anschließend je nach Landnutzung unidirektional dem 2D- 


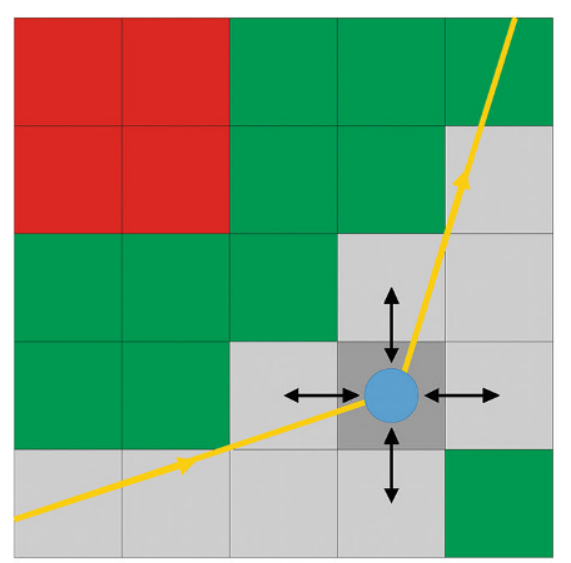

a
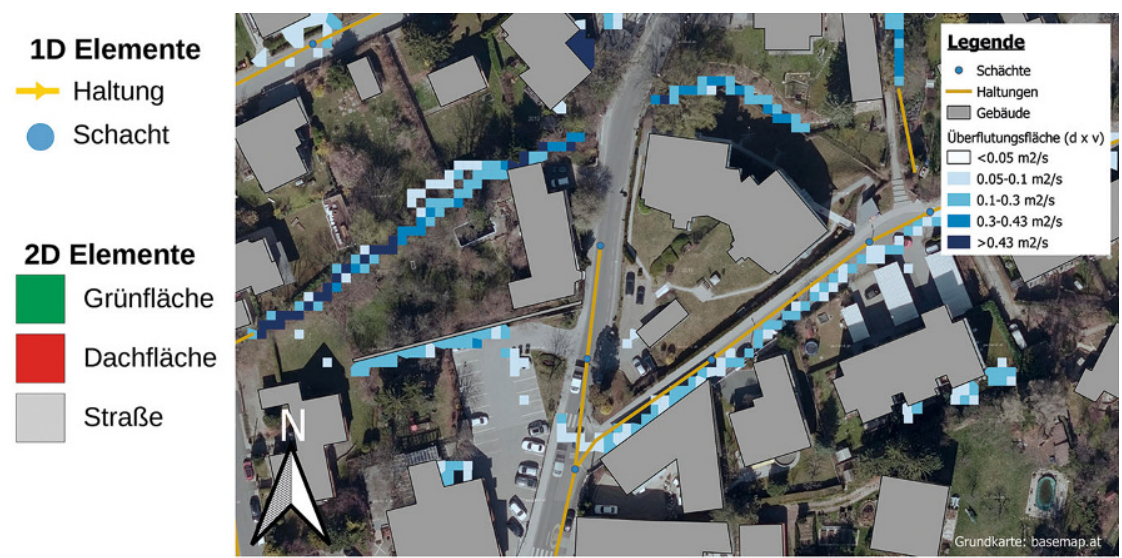

b

Abb. 7 Exemplarische Anwendung des gekoppelten 1D-2D-Ansatzes für eine Überflutungsmodellierung in einem urbanen Einzugsgebiet. a Kopplung eines 1D-Kanalmodells mit einem 2D-Oberflächenabflussmodell (Auflösung des 2D-Rasters anhand der Auflösung der HRUs (2D-Elemente)); b Modellergebnisse eines 1D-2D-Überflutungsmodells mittels Überlagerung der beiden Zielgrößen Wasserstand und Fließgeschwindigkeit

Modell (z. B. bei Grünflächen oder Straßen) oder dem 1D-Modell (z.B. über angeschlossene Dachflächen) übergeben. Abb. 7 stellt exemplarisch die Anwendung eines gekoppelten 1D-2DAnsatzes dar.

Der gekoppelte 1D-2D-Ansatz eignet sich aufgrund seiner hohen Flexibilität für eine Vielzahl von Fragestellungen im Kontext der Überflutungsmodellierung. Besonders für pluviale Überflutungen in urbanen Einzugsgebieten ist dieser Ansatz geeignet, da dieser die hydrologische Abflussbildung, die Ausbreitung des Oberflächenabflusses in 2D und den Kanalabfluss in 1D parallel berechnet. Ebenfalls können fluviale Überflutungsereignisse mit diesem Ansatz modelliert werden (1D-Abfluss im Gerinne mit einer 2D-Ausbreitung des Oberflächenabflusses bei Erreichen der Kapazitätsgrenzen), was vor allem auch die Berücksichtigung von Stadtbächen erlaubt. Durch die eindimensionale Modellierung der Gerinne sind Auswirkungen wie Verklausungen von Durchlässen sehr leicht in Form verkleinerter Querschnitte zu berücksichtigen. Zusätzlich können Wasserstände sowie Zuflüsse aus den Randgebieten (Hangwasser, Hochwasserwelle) in Form von äußeren Randbedingungen in das Modell integriert werden, sodass auch größere Flusseinzugsgebiete sowie Hangeinzugsgebiete im urbanen und ruralen Raum mit akzeptablen Simulationszeiten modelliert werden können. Allerdings ist aufgrund der Kopplung zweier Modelle ein hoher Datenaufwand erforderlich, sodass der Aufbau eines 1D-2D-Überflutungsmodells und die entsprechende Datenaufbereitung einen hohen Zeitaufwand bedeuten können.

\section{Zusammenfassung}

Überflutungen im urbanen Raum haben in den letzten Jahren hohe Aufmerksamkeit erlangt. Während die Analyse von „klassischen“ Flusshochwässern seit langem Gegenstand in Wissenschaft und Praxis ist, ist die Betrachtung von Überflutungen, die außerhalb von Fließgewässern entstehen, ein relativ junges Themenfeld.

In Österreich, aber auch im internationalen Raum, werden teilweise unterschiedliche Begriffe für diese Art von Überflutungen genutzt. Neben der Unterscheidung in fluviale Hochwässer Hochwässer, die aus einem Gewässer heraus entstehen - und pluvialen Hochwässern - Hochwässer, die ohne einen Beitrag aus einem ständig Wasser führenden Gewässer entstehen -, werden für die pluvialen Hochwässer noch weitere Begriffe verwendet.

Dabei werden die Begriffe pluviale Überflutung, Sturzfluten, Hangwasser und Überflutung durch Oberflächenwasser häufig für die gleichen Prozesse benutzt. Im Rahmen einer Überflutungsanalyse ist daher eine exakte Bestimmung und Kommunikation der beteiligten Prozesse notwendig.

Für die Überflutungsanalyse steht mittlerweile eine Vielzahl von Werkzeugen zur Verfügung. In diesem Beitrag wurden die wichtigsten Modellansätze hierarchisch geordnet kurz dargestellt.
Die mittlerweile zur Verfügung stehenden Ansätze umfassen:

- GIS-basierte Methoden zur Bestimmung der Fließwege an der Oberfläche,

- zelluläre Automaten zur Bestimmung von Überflutungsflächen,

- 1D-hydrodynamische Kanalnetzmodelle,

- 2D-hydrodynamische Oberflächenabflussmodelle,

- gekoppelte 1D-2D-Überflutungsmodelle.

Die hierarchische Darstellung der Modellansätze bedeutet dabei keine Wertung der Modelle, vielmehr ist eine der jeweiligen Aufgabe und dem jeweiligen Untersuchungsgebiet angepasste Auswahl der Modellansätze und eine eventuelle Kombination dieser Ansätze anzustreben.

Funding Open access funding provided by Graz University of Technology.

Open Access Dieser Artikel wird unter der Creative Commons Namensnennung 4.0 International Lizenz veröffentlicht, welche die Nutzung, Vervielfältigung, Bearbeitung, Verbreitung und Wiedergabe in jeglichem Medium und Format erlaubt, sofern Sie den/die ursprünglichen Autor(en) und die Quelle ordnungsgemäß nennen, einen Link zur Creative Commons Lizenz beifügen und angeben, ob Änderungen vorgenommen wurden. 
Die in diesem Artikel enthaltenen Bilder und sonstiges Drittmaterial unterliegen ebenfalls der genannten Creative Commons Lizenz, sofern sich aus der Abbildungslegende nichts anderes ergibt. Sofern das betreffende Material nicht unter der genannten Creative
Commons Lizenz steht und die betreffende Handlung nicht nach gesetzlichen Vorschriften erlaubt ist, ist für die oben aufgeführten Weiterverwendungen des Materials die Einwilligung des jeweiligen Rechteinhabers einzuholen.
Weitere Details zur Lizenz entnehmen Sie bitte der Lizenzinformation auf http://creativecommons.org/licenses/ by/4.0/deed.de.

\section{Literatur}

Bates, P. D. and De Roo, A. P. J. (2000): A simple raster-based model for flood inundation simulation. Journal of Hydrology, 236(1-2), 54-77.

Berndtsson, R., Becker, P., Persson, A., Aspegren, H., Haghighatafshar, S., Jönsson, K., Larsson, R., Mobini, S., Mottaghi, M., Nilsson, J., Nordström, J., Pilesjö, P., Scholz, M., Sternudd, C., Sörensen, J., and Tussupova, K. (2019): Drivers of changing urban flood risk: A framework for action. Journal of Environmental Management, 240, 47-56.

Burek, P., Knijff van der, J., Roo de, A., European Commission, Joint Research Centre, and Institute for the Protection and the Security of the Citizen (2013): LISFLOOD, distributed wate balance and flood simulation model: revised user manual 2013., Luxembourg, Publications Office. [online] http://dx.publications.europa eu/10.2788/24719 (Accessed June 30, 2020).

Chifflard, P., Didszun, J., and Zepp, H. (2008): Skalenübergreifende Prozess-Studien zur Abflussbildung in Gebieten mit periglazialen Deckschichten (Sauerland, Deutschland). Grundwasser, 13(1), 27-41.

EC (2007): Richtlinie 2007/60/EG des Europäischen Parlamentes und des Rates vom 23. Oktober 2007 über die Bewertung und das Management von Hochwasserrisiken,

ESRI (2021): Abflussakkumulation - Hilfe ArcGIS for Desktop. [online] http://desktop arcgis.com/de/arcmap/10.3/tools/spatial-ana lyst-toolbox/flow-accumulation.htm (Accessed February 22, 2021).

Fairfield, J. and Leymarie, P. (1991): Drainage networks from grid digital elevation models. Water Resources Research, 27(5), 709-717.

Fan, Y., Ao, T., Yu, H., Huang, G., and Li, X. (2017): A Coupled 1D-2D Hydrodynamic Model for Urban Flood Inundation. Advances in $\mathrm{Me}$ teorology, 2017, e2819308. [online] https://www. hindawi.com/journals/amete/2017/2819308/

(Accessed February 1, 2021).

Finaud-Guyot, P., Delenne, C., Guinot, V., and Llovel, C. (2011): 1D-2D coupling for river flow modeling. Comptes Rendus Mecanique, 339(4), 226-234.

Ghimire, B., Chen, A. S., Guidolin, M., Keedwell, E. C., Djordjevic, S., and Savic, D. (2013): Formulation of a fast 2D urban pluvial flood model using a cellular automata approach. Journal of Hydroinformatics, 15(3), 676-686.

Jamali, B., Bach, P. M., and Deletic, A. (2020): Rainwater harvesting for urban flood management-An integrated modelling framework. Water Research, 171, 115372
Jenson, S. K. and Domingue, J. O. (1988): Extracting Topographic Structur from Digital Elevation Data for Geographic Information System Analysis. Photogrammetric Engineering and remote sensing, Vol.54(No.11), 1593-1600.

Kleidorfer, M., Leimgruber, J., Simperler, L., Zeisl, P., Kretschmer, F., Himmelbauer, P. Krebs, G., Ertl, T., Stöglehner, G., and Muschalla, D. (2019): Endbericht Projekt FLEXADAPT - Langversion inklusive theoretischer Grundlagen, Wien, Österreich, Bundesministerim für Nachhaltigkeit und Tourismus. [online] https:// www.bmlrt.gv.at/service/publikationen/wasser/ Endbericht-Projekt-Felxadapt---Langversioninklusive-theoretischer-Grundlagen.html (Accessed January 26, 2021).

Kron, W., Eichner, J., and Kundzewicz, Z. W. (2019): Reduction of flood risk in Europe-Reflections from a reinsurance perspective. Journal of Hydrology, 576, 197-209.

Leandro, J., Chen, A. S., Djordjevic, S., and Savic, D. A. (2009): Comparison of $1 \mathrm{D} / 1 \mathrm{D}$ and 1D/2D Coupled (Sewer/ Surface) Hydraulic Models for Urban Flood Simulation. Journal of Hydraulic Engineering, 135(June 2009), 495-504. Leavesley, G. H., Lichty, R. W., Troutman, B. M. and Saindon, L. G. (1983): Precipitation-runoff modeling system; user's manual, U.S. Geological Survey, Water Resources Division,. [online http://pubs.er.usgs.gov/publication/wri834238 (Accessed February 1, 2021).

Leimgruber, J., Steffelbauer, D. B., Krebs, G. Tscheikner-Gratl, $\mathrm{F}_{\text {, }}$ and Muschalla, D. (2018): Selecting a series of storm events for a modelbased assessment of combined sewer overflows. Urban Water Journal, 15(5), 453-460.

Löwe, R., Urich, C., Sto. Domingo, N., Mark, O., Deletic, A., and Arnbjerg-Nielsen, K. (2017): Assessment of urban pluvial flood risk and efficiency of adaptation options through simulations-A new generation of urban planning tools. Journa of Hydrology, 550, 355-367.

Mosley, M. P. and McKerchar, A. I. (1993): "Chapter 8: Streamflow" in Handbook of Hydrology.

Muschalla, D., Sulzbacher, R. M., Leimgruber, J., Maier, R., Ertl, T., Neunteufel, R., Kretschmer, F., Kleidorfer, M., and Tscheikner-Gratl, F (2015): Auf effizientem Wege von den Daten zum Modell (DATMOD) - Sanierungs- und Anpassungsplanung von kleinen und mittleren Kanalnetzen, Wien, Österreich, Bundesministerium für Land- und Forstwirtschaft, Umwelt und Wasserwirtschaft. [online] https:// www.umweltfoerderung.at/fileadmin/user
upload/media/umweltfoerderung/Dokumente Betriebe/Wasser_Betriebe/Studien_Wasserwirt schaft/DATMOD_Leitfaden_160217.pdf (Accessed April 6, 2020)

O'Callaghan, J. F. and Mark, D. M. (1984): The extraction of drainage networks from digital elevation data. Computer Vision, Graphics, and Image Processing, 28(3), 323-344.

ÖWAV (2007): ÖWAV-Regelblatt 19 - Richtlinien für die Bemessung von Mischwasserentlastungen. Österreichischer Wasser- und Abfallwirtschaftsverband.

ÖWAV (2009): ÖWAV-Regelblatt 11: Richtlinien für die abwassertechnische Berechnung und Dimensionierung von Abwasserkanälen.

Pasquier, U., He, Y., Hooton, S., Goulden, M. and Hiscock, K. M. (2019): An integrated 1D-2D hydraulic modelling approach to assess the sensitivity of a coastal region to compound flooding hazard under climate change. Natural Hazards, 98(3), 915-937.

Schmitt, T. G., Thomas, M., and Ettrich, N. (2004): Analysis and modeling of flooding in urban drainage systems. Journal of Hydrology, 299(3), 300-311.

Sene, K. (2015): Flash floods: forecasting and warning., Springer

Sitterson, J., Parmar, R., Wolfe, K., Muche, M. and Avant, B. (2017): An Overview of RainfallRunoff Model Types, Washington D.C., USA, United States Environmental Protection Agency.

Winsemius, H. C., Aerts, J. C. J. H., van Beek, L. P. H., Bierkens, M. F. P., Bouwman, A., Jongman, B., Kwadijk, J. C. J., Ligtvoet, W., Lucas, P. L., van Vuuren, D. P., and Ward, P. J. (2016): Global drivers of future river flood risk. Nature Climate Change, 6(4), 381-385.

Zahnt, N., Eder, M., and Habersack, H. (2018): Herausforderungen durch pluviale Überflutungen - Grundlagen, Schäden und Lösungsansätze. Österreichische Wasser- und Abfallwirtschaft, 70(1-2), 64-77.

Zekâi, S. (2017): Flood modeling, prediction and mitigation, New York, NY, Springer Berlin Heidelberg.

Hinweis des Verlags Der Verlag bleibt in Hinblick auf geografische Zuordnungen und Gebietsbezeichnungen in veröffentlichten Karten und Institutsadressen neutral. 